\title{
Ruptured Mycotic Superior Mesenteric Artery Aneurysms and Infective Endocarditis
}

\author{
Hentati Rim ${ }^{1}$, Tlili Rami², Azaiez Fares², Zouari Fatma², Zayed Soufien ${ }^{1}$, Ben Romdhane Rim², Ben Ameur \\ Youssef $^{2}$
}

Mongi Slim La Marsa Hospital; Faculty of Medicine, ${ }^{1}$ University of Sfax, Tunisia and ${ }^{2}$ University of Tunis El-Manar, Tunis, Tunisia.

\section{Corresponding Author: \\ Dr. Hentati Rim \\ Email: hentati68@yahoo.fr}

This is an Open Access article distributed under the terms of the Creative Commons Attribution License (creativecommons.org) licenses/by/3.0).

$\begin{array}{lll}\text { Received } & : & \text { May 24, } 2019 \\ \text { Accepted } & : & \text { July 20, 2019 } \\ \text { Published } & : & \text { August 5, 2019 }\end{array}$

\begin{abstract}
Background: Aneurysms of the superior mesenteric artery (SMA) and its branches are uncommon complication of infective endocarditis (IE) and have a high rate of rupture and mortality. Case Report: A 50-year-old female was diagnosed and treated for IE with good initial evolution. During pre-surgery evaluation, the patient developed hemorrhagic shock. Computed tomography angiogram revealed hemoperitoneum and contrast extravasations due to rupture of aneurysm originating from an SMA's branch. The patient was transferred for urgent surgery. On laparotomy large, inflamed, pulsatile mesenteric mass with free blood in the pelvis was found. Evidence of necrotic small bowel was detected and resection of $25 \mathrm{~cm}$ with ileostomy was performed and surgery for restoring bowel continuity after ileostomy was programmed three months later with good results. Conclusion: Although main trunk and superior mesenteric artery (SMA) branch aneurysms account for only $5.5 \%$ of all visceral artery aneurysms, early identification and expedient management are very important given an associated $38-50 \%$ rupture rate and $30 \%$ mortality.
\end{abstract}

Keywords: Aneurysm, Angiography, Endocarditis, Ileostomy, Superior Mesenteric Artery.

\section{Introduction}

Infective endocarditis (IE) is associated with high mortality and severe complications. Infectious aneurysms, a rare complication, result from septic arterial embolism to the intra-luminal space or vasa vasorum or from subsequent spread of infection through the intimal vessels. Aneurysms of the superior mesenteric artery (SMA) and its branches are uncommon and have a high rate of rupture and mortality. We describe the presentation and management of a case of infective endocarditis and ruptured SMA branch aneurysm.

\section{Case Report}

A 50-year-old female, diagnosed with mitral insufficiency 15 years ago presented with fever associated with systemic symptoms of chills, poor appetite and weight loss. Physical examination showed a systolic murmur at mitral region. Transthoracic echocardiography showed valvular vegetation $(15 \times 10 \mathrm{~mm})$ associated with mild mitral insufficiency. The patient was treated with probabilistic antibiotic therapy (oxacillin, ampicillin and gentamycin) with good initial evolution. During pre-surgery evaluation, the patient developed abdominal pain, and hemorrhagic shock. Computed tomography angiogram revealed hemoperitoneum, and contrast extravasations due to aneurysms rupture originating from an SMA's branch [Fig.1]. The patient was transferred for urgent surgery. On laparotomy large, inflamed, pulsatile mesenteric mass with free blood in the pelvis was found. The transverse mesocolon was retracted and proximal control of the SMA obtained at the base of the mesentery. The aneurysm was 


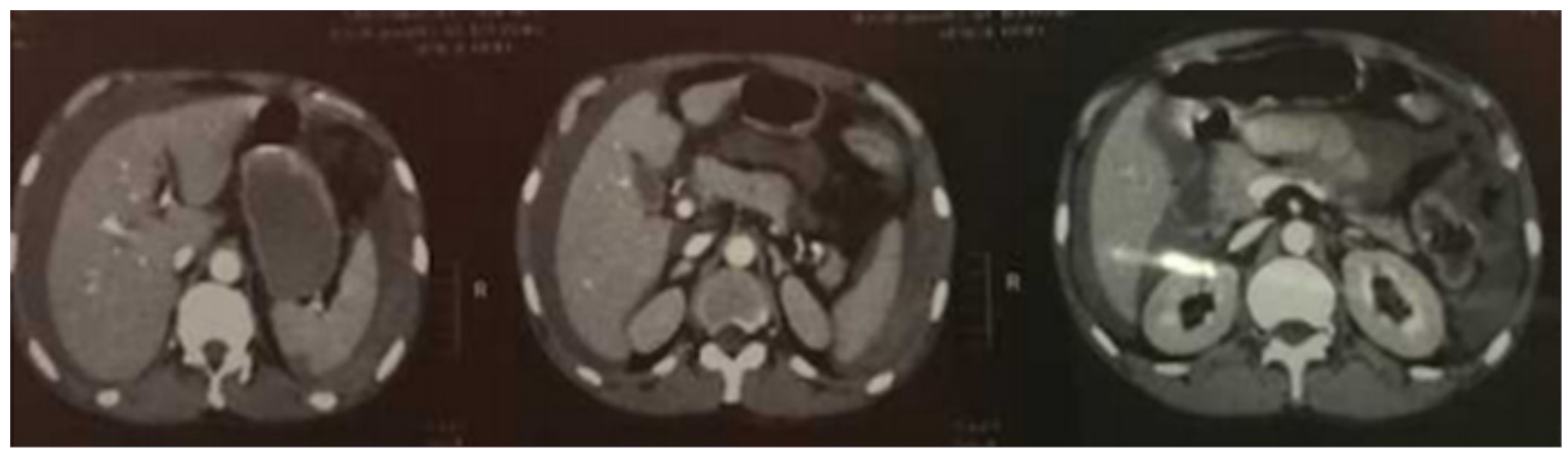

Fig.1: Computed tomography image of an infected aneurysm of intestinal branch of the superior mesenteric artery. Hemoperitoneum, and contrast extravasation are visible.

adherent to the retroperitoneum. Evidence of necrotic small bowel was detected and resection of $25 \mathrm{~cm}$ with ileostomy was performed [Fig.2]. The entire bowel was inspected and showed no evidence of further ischemia. On $6^{\text {th }}$ postoperative day (POD), the patient was extubated and transferred out of the intensive care. Although cultures of the mesenteric aneurysm were sterile, urine culture drawn after the surgery revealed Klebsiella pneumonia BLSE (bêta-lactamases à spectre étendu). After a 49-day hospital stay, the patient was discharged and a surgery for restoring bowel continuity after ileostomy was programmed three months later with good results.

\section{Discussion}

SMA aneurysms are a rare complication of infective endocarditis. In fact, the true incidence of main trunk SMA aneurysms is unknown but they are estimated to number between 5.5-8.6\% of all splanchnic artery aneurysms. Side branch aneurysms are even rarer, as did our patient [1-8]. Incidence and rupture risk are as much as twofold higher in men and 19\% of patients will have synchronous aneurysmal disease at other sites $[5,6]$. The number of SMA aneurysms discovered incidentally, with the utilization of cross-sectional imaging, has risen from $10 \%$ to $50 \%$ in some studies [4,6,9-11]. Reported size for main SMA trunk aneurysms varies from 2 to $9 \mathrm{~cm}$, with side

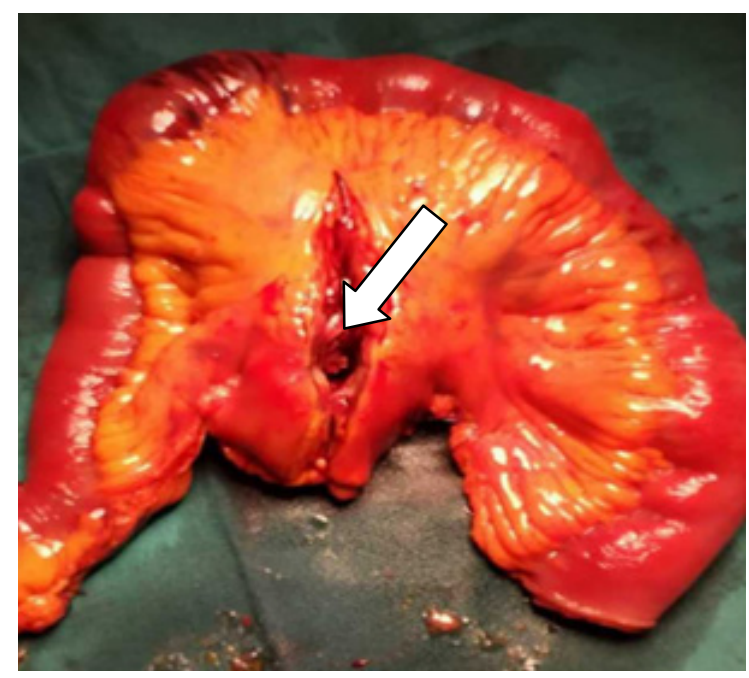

Fig.2: Intestinal resection surgical specimen. The arrow shows the mycotic aneurysms of SMA branch.

branch aneurysms usually less than $1 \mathrm{~cm}[12,13]$. Historically, infection has been the major etiology of SMA aneurysms, accounting for 31 to $63 \%$ of them $[13,14]$. The microorganisms mostly implicated are Streptococcal and Staphylococcal species; however blood cultures are negative in 18 to $50 \%$ of cases and failure to isolate specific microbes from excised tissue is not uncommon [6,15]. This was the case in our patient. Nevertheless, a germ was identified in his urine culture which causality can't be assured.

The most common symptom associated with SMA aneurysms is abdominal or back pain, with a 
frequency of 60 to $70 \%$ of patients $[5-8,11]$. Rupture risk is higher than other splanchnic aneurysms (38\% to $50 \%)$. It can manifest either by intraperitoneal hemorrhage, mesenteric hematoma, or intra-luminal rupture with gastrointestinal bleeding $[5,6,8]$. The correlation between aneurysm size and rupture risk has not been completely established. Conventional arteriography was used to diagnose up to $75 \%$ of SMA aneurysms. It has now been supplanted by cross-sectional imaging [5].

Endovascular techniques, ranging from trans-catheter embolization to stent grafting, have been used to treat SMA aneurysms, even in the setting of rupture with hemodynamic instability $[1,4,6,7,9]$. These techniques offer advantages by virtue of their minimally invasive nature and potential for preservation of in-line flow. However, there are obvious concerns surrounding their adoption for the management of mycotic aneurysms. In fact, when endovascular strategies were used at other anatomic sites, the rate of persistent infection was as high as $25 \%$, and 1 -year survival was as low as $39 \%$ in reported series [15]. Concomitant bowel resection has been required in more than one-third of cases in some of the larger series of SMA aneurysms, thus, attentive evaluation to rule out potential bowel ischemia is imperative before the decision of an endovascular approach [6]. Endovascular treatment for mycotic SMA and branch aneurysms, may have retain a place in patients with high operative risk and limited life expectancy. Open surgical repair remains the preferred option for management of infected SMA aneurysms. The typical approach is trans-peritoneal. Given well-developed collateral flow, resection without reconstruction usually suffices $[6,12]$ and branch aneurysms can frequently be treated with simple ligation. Per and post-operative vigilance for proof of intestinal ischemia is mandatory, and reconstruction is more likely to be necessary for proximal main trunk aneurysms $[3,8]$.

\section{Conclusion}

Endovascular options may be suitable in some cases, but open surgical resection with or without reconstruction remains the standard treatment for infected aneurysms.

Contributors: HR: manuscript writing, patient management; TR, AF, ZF: manuscript editing, patient management; ZS, BRR, BAY: critical inputs into the manuscript. HR will act as a study guarantor. All authors approved the final version of this manuscript.

Funding: None; Competing interests: None stated.

\section{References}

1. Drescher R, Köster O, von Rothenburg T. Superior mesenteric artery aneurysm stent graft. Abdom Imaging. 2006;31(1):113-116.

2. Friedman SG, Pogo GJ, Moccio CG. Mycotic aneurysm of the superior mesenteric artery. J Vasc Surg. 1987;6(1):87-90.

3. Ando M, Ito M, Mishima Y. Spontaneous dissecting aneurysm of the main trunk of the superior mesenteric artery: report of a case. Surg Today. 1995;25(5):468-470.

4. Carr SC, Pearce WH, Vogelzang RL, McCarthy WJ, Nemcek AA, Yao JS. Current management of visceral artery aneurysms. Surgery. 1996;120(4):627-633; discussion 633-634.

5. Shanley CJ, Shah NL, Messina LM. Uncommon splanchnic artery aneurysms: pancreaticoduodenal, gastroduodenal, superior mesenteric, inferior mesenteric, and colic. Ann Vasc Surg. 1996;10(5):506-515.

6. Stone WM, Abbas M, Cherry KJ, Fowl RJ, Gloviczki P. Superior mesenteric artery aneurysms: Is presence an indication for intervention? J Vasc Surg. 2002;36(2):234-237.

7. Schweigert M, Adamus R, Stadlhuber RJ, Stein HJ. Endovascular stent--graft repair of a symptomatic superior mesenteric artery aneurysm. Ann Vasc Surg. 2011;25(6):841.e5-8.

8. Zimmerman-Klima PM, Wixon CL, Bogey WM, Lalikos JF, Powell CS. Considerations in the management of aneurysms of the superior mesenteric artery. Ann Vasc Surg. 2000;14(4):410-414.

9. Sachdev U, Baril DT, Ellozy SH, Lookstein RA, Silverberg D, Jacobs TS, et al. Management of aneurysms involving branches of the celiac and superior mesenteric arteries: a comparison of surgical and endovascular therapy. J Vasc Surg. 2006;44(4):718-724. 
10. Berceli SA. Hepatic and splenic artery aneurysms. Semin Vasc Surg. 2005;18(4):196-201.

11. Saltzberg SS, Maldonado TS, Lamparello PJ, Cayne NS, Nalbandian MM, Rosen RJ, et al. Is endovascular therapy the preferred treatment for all visceral artery aneurysms? Ann Vasc Surg. 2005;19(4):507-515.

12. Buchs NC, Skala K, Sierra J, Huber O, Morel P. Mycotic aneurysm of the superior mesenteric artery. Surgery. 2013;153(1):133-134.
13. Stanley JC, Thompson NW, Fry WJ. Splanchnic artery aneurysms. Arch Surg. 1970;101(6):689-697.

14. Graham JM, McCollum CH, DeBakey ME. Aneurysms of the splanchnic arteries. Am J Surg. 1980;140(6):797.

15. Lee W-K, Mossop PJ, Little AF, Fitt GJ, Vrazas JI, Hoang JK, et al. Infected (mycotic) aneurysms: spectrum of imaging appearances and management. Radiogr Rev Publ Radiol Soc N Am Inc. 2008;28(7):1853-1868. 